\title{
1 13-valent vaccine serotype pneumococcal community acquired 2 pneumonia in adults in high clinical risk groups
}

4 Priya Daniel ${ }^{1}$, Chamira Rodrigo $^{1}$, Thomas Bewick ${ }^{2}$, Carmen Sheppard ${ }^{3}$, Sonia Greenwood ${ }^{2}$, Tricia $5 \quad$ M McKeever $^{4}$, Caroline Trotter ${ }^{5}$, Wei Shen Lim $^{1}$

\section{Correspondence}

$8 \quad$ P Daniel

9 Department of Respiratory Medicine, David Evans Building

10 City Hospital Campus, Nottingham University Hospitals NHS Trust

11 Hucknall Road, Nottingham. NG5 1PB

12 Email: priyasosha.daniel@nuh.nhs.uk

13 Tel: +44(0)1159691169

\section{Affiliations}

$16{ }^{1}$ Department of Respiratory Medicine, Nottingham University Hospitals NHS Trust, Nottingham

17 NG5 1PB, UK;

$18{ }^{2}$ Department of Respiratory Medicine, Derby Teaching Hospitals NHS Foundation Trust, Derby 19 DE22 3NE, UK;

203 Respiratory and Vaccine Preventable Bacteria Reference Unit, Public Health England, 21 Microbiology Services Division, Colindale Avenue, London NW9 5EQ, UK;

$22{ }^{4}$ Division of Epidemiology, University of Nottingham, Clinical Skills Building, Nottingham City 23 Hospital campus, Hucknall Road, Nottingham NG5 1PB, UK;

245 Disease Dynamics Unit, Department of Veterinary Medicine, University of Cambridge, 25 Madingley Road, Cambridge CB3 OES, UK; 


\section{Keywords}

27 Streptococcus pneumoniae; pneumonia; PCV-13, serotypes; vaccine

28

29 Word count, references, tables and figures

30 Abstract: 237

31 Main text: 3102 words

32 References: 40

33 Tables: 4

34 Figures: 2 


\section{13-valent vaccine serotype pneumococcal community acquired}

36 pneumonia in adults in high clinical risk groups

37 Priya Daniel, Chamira Rodrigo, Thomas Bewick, Carmen Sheppard, Tricia M McKeever, Sonia 38 Greenwood, Caroline Trotter, Wei Shen Lim

40 Abstract

41 There is debate regarding the value of vaccinating adults with the 13-valent pneumococcal

42 conjugate vaccine (PCV-13). This analysis was conducted to investigate the risk of PCV-13

43 serotype community acquired pneumonia (CAP) in hospitalised adults with co-morbid disease 44 and risk factors for pneumococcal disease in the UK.

46 Consecutive adults hospitalised (2008 - 2013) with a primary diagnosis of CAP, were recruited.

47 Pneumococcal aetiology disease was identified by use of pneumococcal urinary antigen 48 detection and serotype identification using a validated multiplex immunoassay or serum latex 49 agglutination. Adults with PCV-13 serotype CAP were compared to those with non-PCV-13 50 serotype CAP.

52 Of 2224 patients, PCV-13 serotype CAP was identified in 337 (15.2\%) and non-PCV-13 serotype

53 CAP in 250 (11.2\%) individuals. Adults aged $\geq 65$ years with one or more clinical risk factors had a 54 significantly lower risk of PCV-13 serotype CAP compared to those aged 16-64 years without 55 clinical risk factors (aOR 0.61, 95\% Cl 0.41-0.92, p=0.018). In a stacked-risk analysis, the presence 56 of incremental clinical risk factors was associated with lower odds of PCV-13 disease ( $p$ for trend 
$57=0.029)$ Adults with underlying chronic respiratory disease (aOR) $0.56,95 \% \mathrm{Cl} 0.36-0.85$,

$58 \mathrm{p}=0.007$ ) and chronic kidney disease (aOR 0.48, 95\% Cl 0.25-0.92, $\mathrm{p}=0.028$ ) had significantly lower

59 adjusted odds of PCV-13 compared to non-PCV-13 serotype CAP.

60

61 This analysis suggests that in the UK, the burden of PCV13 disease is greater in adults outside the

62 traditional 'at-risk' groups compared to adults in 'at-risk' groups.

63 


\section{Introduction}

65 Increasing age and the presence of co-morbid diseases are recognised risk factors for

66 pneumococcal disease..$^{1-4}$ In addition, pneumococcal attributable mortality is higher in these

67 clinical risk groups. 5 Therefore, implementation of appropriate vaccination strategies is

68 important for these individuals. The current UK vaccination policy recommends 23-valent

69 polysaccharide pneumococcal vaccination (PPV-23) in adults at high risk of pneumococcal

70 disease, comprising (a) adults aged between 16-64 years with certain co-morbid diseases, and (b)

71 adults aged 65 years and over. ${ }^{7}$ However, polysaccharide vaccine effectiveness in these risk

72 groups is debated. ${ }^{8-12}$ Immunogenicity studies have shown higher antibody concentrations and

73 functional antibody responses to pneumococcal conjugate compared with polysaccharide

74 vaccination in adults at higher risk of pneumococcal disease including those with human

75 immunodeficiency virus (HIV), chronic obstructive pulmonary disease and older adults. ${ }^{13-15}$

76 Therefore, such patients may benefit from the administration of pneumococcal conjugate

77 vaccination (PCV) in addition to, or in place of the current polysaccharide vaccine. In randomised

78 controlled trials in Malawi and the Netherlands, administration of the pneumococcal conjugate

79 vaccine reduced vaccine-type (VT) invasive pneumococcal disease (IPD) and community acquired

80 pneumonia (CAP) in risk groups of immunocompromised adults with HIV and those over the age

81 of 65 years, respectively. ${ }^{16} 17$ However, any assessment of the benefits of vaccinating adults with

82 the conjugate vaccine needs to take into account the burden of VT disease in the target group.

83 In the UK, there has been a substantial decrease in adult pneumococcal VT disease as a

84 consequence of herd protection following the introduction of the infant pneumococcal 85 vaccination programme; this decrease is apparent for both invasive and non-invasive 
86 pneumococcal disease. ${ }^{18-21}$ In patients with IPD, these herd effects appear similar among patients

87 with and without clinical risk factors for pneumococcal disease. ${ }^{3}$ There are no such relevant data

88 in adults with non-invasive pneumococcal pneumonia.

89

90 In this study, we sought to determine whether hospitalised individuals at high risk of

91 pneumococcal disease are more likely to have PCV-13 serotype CAP compared to non-PCV-13

92 serotype CAP. 


\section{$93 \underline{\text { Methods }}$}

\section{Study design}

95 We conducted a prospective cohort study of consecutive adult patients admitted, with a primary

96 diagnosis of community acquired pneumonia, to two large university hospitals in Nottingham,

97 between September 2008 and 2013. Combined, these two hospitals cover the catchment area

98 for acute and emergency admissions in the Greater Nottingham area. All patients admitted to

99 medical admissions units were screened every weekday, using radiological and clinical records,

100 to assess for study eligibility. Study eligible patients were aged 16 years or over, presenting with

101 symptoms of a lower respiratory tract infection (at least one of: cough, increasing breathlessness,

102 sputum production and fever), who had radiographic infiltrates consistent with respiratory

103 infection, and who were treated by their clinical team for a diagnosis of CAP. Adults hospitalised

104 in the 10 days preceding the index admission or who had a diagnosis of tuberculosis or post-

105 obstructive pneumonia were excluded. Informed consent was obtained from all study patients;

106 in the event that patients lacked capacity, patient personal consultees were approached for proxy

107 consent. Patient demographics and clinical details were collected from patient records. All study 108 procedures were approved by Nottingham Research Ethics Committee.

\section{Study population}

111 Routine microbiological investigations were performed at the discretion of the clinical team. In

112 addition, urine samples were taken on admission from each individual for pneumococcal specific

113 microbiological analysis; Binax-NOW ${ }^{\circledR}$ assays were performed for pneumococcal C- 
114 polysaccharide urinary antigen detection (UAD) at the local microbiological laboratories whilst

115 the remaining volume of urine was frozen and batch transported to Public Health England (PHE)'s

116 Respiratory and Vaccine Preventable Bacteria Reference Unit in Colindale for serotyping of

117 pneumococcal strains by a multiplex immunoassay (Bio-plex). The Bio-plex assay was validated

118 for detection of pneumococcal serotypes $1,3,4,5,6 \mathrm{~A} / \mathrm{C}, 6 \mathrm{~B}, 7 \mathrm{~F} / \mathrm{A}, 8,9 \mathrm{~V}, 14,18,19 \mathrm{~A}, 19 \mathrm{~F}$ and

$11923 \mathrm{~F} .{ }^{22}$ The sensitivity and specificity for pneumococcal detection using the Binax-NOW ${ }^{\circledR}$ method,

120 is $74 \%$ and $97 \%$, respectively and for the Bio-plex method, is $79 \%$ and $99 \%$, respectively. 2223

121 Bacteraemic cases of CAP due to Streptococcus pneumoniae were identified and serotyped by

122 serum latex agglutination at PHE's reference laboratory. Patients were considered to have

123 pneumococcal CAP if any of the following criteria were met: (a) a positive pneumococcal UAD, or

124 (b) a positive blood culture for $S$ pneumoniae, or (c) pneumococcal serotype detection by the Bio125 plex assay.

126

\section{Statistical considerations}

128 Statistical analyses were performed using Stata/IC 13.1 (OStataCorp., 2013). Serotypes were 129 grouped into PCV-7 types (serotypes 4, 6B, 9V, 14, 18C, 19F and 23F), 'additional' PCV-13 types 130 not present in PCV-7 (serotypes 1, 3, 5, 6A/C, 7F/A, 19A) and 'other' non-PCV-13 serotypes. PCV-

13113 disease was defined as the identification of one or more of serotypes in either the PCV-7 or

132 'additional' PCV-13 groups. Non-PCV-13 disease was defined as the isolation of any other 133 pneumococcal serotype or the presence of 'untyped' non-invasive pneumococcal CAP (based on

134 a positive UAD). Baseline characteristics and putative co-morbid disease risk factors for PCV-13

135 disease were compared using Pearson's chi-square or Fisher's tests for categorical variables, and 
136 the Mann Whitney U-test for non-parametric continuous variables. The independent association

137 between baseline co-morbidity and PCV-13 disease compared to non-PCV-13 disease was

138 examined using a multivariable logistical regression model; those co-morbid diseases with a $p$

139 value of $<0.2$ on univariate analysis were included in the multivariable model. Likelihood ratio

140 tests were used to determine the best model fit for continuous variables. Secondary analysis

141 were conducted examining the odds of PCV-13 disease in (a) all 'at-risk' individuals (defined as

142 those aged 16-64 with a clinical risk factor for pneumococcal disease or those $\geq 65$ years), (b)

143 individuals stratified according to age (dichotomised at 65 years) and the presence of a clinical

144 risk factor for pneumococcal disease: (1) aged 16-64 years without a clinical risk factor, (2) aged

145 16-64 years with one or more clinical risk factors, (3) aged $\geq 65$ years without a clinical risk factor,

146 (4) aged $\geq 65$ years with one or more clinical risk factors and (c) individuals with increasing

147 numbers of clinical risk factors; gender was included a priori in these models. Clinical risk factors

148 for pneumococcal disease were defined as those eligible for pneumococcal vaccination in the UK

149 as described in PHE's 'Immunisation against Infectious Diseases'; in brief, risk factors included

150 chronic respiratory disease, chronic heart disease, chronic kidney disease, chronic liver disease,

151 immunosuppression, diabetes, splenic dysfunction and individuals with cerebrospinal fluid (CSF)

152 leaks or cochlear implants. ${ }^{7}$ Immunosuppression was defined as the presence of splenic

153 dysfunction, haematological disease including malignancy, solid organ or bone marrow

154 transplant, immunodeficiency, treatment with immunosuppressive medication (not including

155 steroids) or HIV; all other case definitions were derived from a previous study examining clinical

156 risk groups in pneumococcal disease. ${ }^{24}$ 
158 Incidence data for pneumococcal CAP in the Greater Nottingham area were calculated using data 159 on population demographics collected from (a) the National Infection Service, PHE, for adults 160 aged 16-64 with clinical risk factors, and (b) the UK census (2011) for adults aged $\geq 65$ years. ${ }^{25}$

161 As there is no national registry of risk groups for pneumococcal disease, population demographic 162 data for influenza risk groups were taken as a surrogate measure for incidence calculations. ${ }^{26}$ 163 


\section{Results}

\section{Study population}

166 Over the 5 year study period, 2702 patients were eligible for study inclusion. Of these, 284

167 (10.5\%) were subsequently found to have an alternative diagnosis to CAP and in a further 194

168 patients, study consent was not obtained. The final study cohort consisted of 2224 adults.

169 Patients in whom consent was not obtained were older (median age: 82 years, IQR 73-89 years

170 versus 71 years, IQR 56-80 years, $p<0.001$ ) and were more likely to have chronic kidney disease

$171(13.4 \%$ versus $7.6 \%, p=0.004)$, cerebrovascular disease $(21.8 \%$ versus $9.1 \%, p<0.001)$ and

172 dementia (33.5\% versus $2.0 \%, p<0.001)$ compared to patients in the study cohort. They were also

173 less likely to have chronic respiratory disease ( $14.4 \%$ versus $25.7 \%, p<0.001)$. No other biases in

174 co-morbid diseases were observed.

175 Pneumococcal CAP was diagnosed in 643 of 2224 (28.9\%) individuals. Urine was unavailable for

176 serotype analysis in $56(8.7 \%)$ of 643 cases. One or more serotypes were identified in $429(66.7 \%)$

177 of 643 cases of pneumococcal CAP; the remainder represent untyped cases of pneumococcal

178 CAP. Cases where urine was unavailable for serotyping were excluded from analysis of the

179 association of clinical risk group and PCV-13 disease.

\section{Baseline characteristics}

182 Of 643 patients with pneumococcal CAP, 294 (45.7\%) had one or more clinical risk factors for

183 pneumococcal disease; of these, chronic respiratory disease ( $n=130,44.2 \%$ ) and chronic heart

184 disease $(n=124,42.2 \%)$ represented the majority of cases. There were 68 patients $(10.6 \%)$ aged 
185 16-64 years with a clinical risk factor and 377 (58.6\%) patients were aged $\geq 65$ years. Three

186 hundred and forty nine (54.3\%) patients with pneumococcal disease had no underlying clinical

187 risk factor for pneumococcal disease; one clinical risk factor was present in 205 (31.9\%) patients,

188 two clinical risk factors were present in 62 (9.6\%) patients and three or more clinical risk factors

189 were present in 27 (4.2\%). Diabetes (9.8\%) and chronic respiratory disease (8.7\%) represented

190 the most common co-morbid diseases amongst patients aged 16-64 years, whilst chronic heart

191 disease (29.2\%) and chronic respiratory disease (28.4\%) were the most common co-morbid

192 diseases amongst those aged $\geq 65$ years (Table 1 ). There were no patients with cochlear implants

193 or CSF fluid leaks.

194

195 Clinical risk groups and PCV-13 disease

196 Of 587 pneumococcal CAP cases where a urine was available for serotype identification, PCV-13

197 and non-PCV-13 disease comprised 337 (57.4\%) and 250 (42.6\%) cases respectively. Baseline

198 characteristics of patients with PCV-13 and non-PCV-13 serotype CAP are shown in (Table 2).

199 Patients with underlying chronic respiratory disease and chronic kidney disease had significantly

200 lower odds of PCV-13 disease compared to non-PCV-13 disease (adjusted Odds Ratio (aOR) 0.56,

$20195 \% \mathrm{Cl} 0.36-0.85, \mathrm{p}=0.007$, and aOR 0.48, 95\% Cl 0.25-0.92, p=0.028, respectively). Conversely,

202 those with dementia had significantly higher odds of PCV-13 disease (aOR 3.91, 95\%Cl 1.10-

203 13.91, $p=0.036)$.

204

205 Of patients with pneumococcal CAP, 184 (31.4\%) were aged 16-64 years with no clinical risk 206 factors, 57 (9.7\%) were aged 16-64 years with one or more clinical risk factors, $133(22.7 \%)$ were 
207 aged $\geq 65$ years with no clinical risk factors and $213(36.3 \%)$ were aged $\geq 65$ years with one or

208 more clinical risk factors. In the gender-adjusted model, patients aged $\geq 65$ years with one or

209 more clinical risk factors had a significantly lower risk of PCV-13 serotype CAP compared to those

210 aged 16-64 years without clinical risk factors (aOR 0.61, 95\%Cl 0.41-0.92, p=0.018) (Table 3). In a

211 stacked-risk analysis adjusted for gender and age, the presence of incremental clinical risk factors

212 was associated with lower odds of PCV-13 disease (Figure 1). The gender-adjusted odds of PCV-

21313 disease was lower in patients that comprised the total 'at-risk' group (those aged 16-64 years

214 with clinical risk factors or those aged $\geq 65$ years): aOR $0.71,95 \% \mathrm{Cl} 0.49-1.02, \mathrm{p}=0.062$.

\section{Serotype distribution of pneumococcal CAP by risk group}

217 Table 4 shows the distribution of single serotypes with $>10$ isolates. Serotypes 7F/A and 8 were

218 the most common, both being isolated in 69 patients. Using serotype 8 as reference, serotypes

2193,5 and 14 were significantly associated with causing disease in 'at-risk' patients compared to

220 those not at-risk whilst serotype 7F/A was associated with lower odds of disease in 'at-risk'

221 patients.

222

\section{Incidence of pneumococcal CAP in clinical risk groups}

224 The overall incidence of pneumococcal CAP was 20.7 per 100, 000 persons whilst that of PCV-13

225 CAP was 10.8 per 100,000 and non-PCV-13 CAP was 8.0 per 100,000. The highest overall

226 incidence of pneumococcal CAP was observed in those over 65 years (64.3 per 100,00); in these

227 patients the incidence of PCV-13 serotype CAP was 32.9 per 100,000 persons, and that of non- 
228 PCV-13 serotype CAP was 26.1per 100,000 persons. The incidence of PCV-13 and non-PCV-13

229 pneumococcal CAP by clinical risk group is shown in Figure 2. Incidence rates of non-PCV-13

230 serotype CAP was two to three fold that of PCV-13 serotype CAP in patients aged 16-64 years

231 with chronic liver disease and those who were immunocompromised. Conversely, patients aged

232 16-64 years with diabetes had a higher incidence of PCV-13 compared to non-PCV-13 serotype

233 CAP (16.7 versus 5.6, per 100,000 persons).

234

235 Mortality

236 Overall, 30-day mortality in patients with pneumococcal CAP was $7.5 \%$. Of those individuals 'at-

237 risk' of pneumococcal disease, 30-day pneumococcal CAP mortality was $10.3 \%$ compared to $1.0 \%$

238 in those under 65 years considered not at risk. The highest pneumococcal CAP mortality was

239 observed in individuals $\geq 65$ years with a clinical risk factor ( $14.2 \%)$, followed by those $\geq 65$ years

240 without a clinical risk factor (8.6\%). For those under 65 years with a clinical risk factor, 30-day

241 mortality was $1.5 \%$. There was no significant difference in 30-day mortality in all individuals with

242 PCV-13 compared to non-PCV-13 serotype CAP (8.3\% vs 7.6\%; OR 1.10, 95\% Cl 0.60-2.02,

$243 \mathrm{p}=0.755)$, nor in those individuals classified as 'at-risk' (11.7\% vs $10.5 \%$; OR 1.13, 95\% $\mathrm{Cl} 0.60$ -

$2442.12, p=0.701)$.

245 


\section{Discussion}

247 In adults hospitalised with pneumococcal CAP, we found that PCV-13 serotype CAP was $44 \%$ less

248 likely in patients with chronic respiratory disease and 52\% less likely in chronic kidney disease 249 compared to non-PCV-13 serotype CAP. The odds of PCV-13 serotype CAP were significantly

250 lower with increasing numbers of clinical risk factors for pneumococcal infection.

252 These results are consistent with findings observed from studies in adult IPD, where PCV-13

253 serotypes have been shown to be less frequently associated with the presence of underlying co-

254 morbid disease, compared to non-PCV-13 serotypes. 52728 Similarly, a 16-year cohort study

255 demonstrated that individuals with chronic respiratory disease and chronic kidney disease were

256 more likely to have non-vaccine type (NVT) (non-PCV-13 and non-PPV-23) IPD compared to PCV-

25713 disease. ${ }^{28}$ However, in contrast to analyses in IPD cohorts which have shown an association

258 with younger age and PCV-13 serotype disease, we observed no association between PCV-13

259 serotype disease and age. ${ }^{528}$

261 In the UK, introduction of the infant vaccination programme has been highly successful in

262 reducing VT serotype IPD as a consequence of herd protection. 182930 Whether these reductions

263 in VT disease equally apply to adults at clinical risk of pneumococcal disease as to other adults is

264 less well defined. 313233 In addition, despite overall decreases in VT pneumococcal disease, 265 increases in NVT IPD have been observed in at risk populations including the 266 immunocompromised and those over 65 years. ${ }^{20} 2834$ Our findings, involving mainly adults with 
267 non-invasive CAP, adds to the evidence base that older adults with clinical risk factors are more

268 likely to have non-PCV-13 serotype CAP compared to PCV-13 serotype CAP. Differences in the 269 invasive potential of pneumococcal serotypes may provide a possible explanation for these

270 observations; non-PCV-13 serotypes are generally less invasive compared to PCV-13 serotypes. ${ }^{27}$

271 Consequently, non-PCV-13 serotypes may be more likely to act as opportunistic pathogens in

272 older patients with co-morbid diseases. ${ }^{35-38}$

273

274 Our finding that patients with dementia were much more likely to be hospitalised with CAP due

275 to PCV-13 serotypes was dominated by patients with dementia who had PCV-7 serotype disease

276 (11 of 19 patients) identified in the first 2 years of the study. Whilst social isolation and lack of

277 child contact in patients with dementia might explain this finding, confirmation of this association

278 in a different patient cohort is necessary. ${ }^{39}$

280 In adults under 65 years, the presence of chronic liver disease or immunocompromise were

281 associated with the highest incidence of pneumococcal CAP. Van Hoek et al linked UK IPD cases

282 to Hospital Episode Statistics (HES) data to estimate incidence rates for clinical risk groups; they

283 too demonstrated that the highest incidence of pneumococcal disease in this age group occurred

284 in these conditions. ${ }^{3}$ The absolute incidence rates for each clinical risk factor were considerably

285 lower in our study compared to that of Van Hoek et al and two similar population based IPD

286 studies conducted in Finland and the Netherlands. ${ }^{3626}$ Possible reasons for this difference include

287 (a) the inclusion of infants and children in previous IPD studies and (b) incomplete recruitment

288 of adults in certain high risk groups, to the current study. 


\section{Strengths and limitations of this analysis}

291 To our knowledge this is the first report to describe the relationship between clinical risk factors

292 for pneumococcal disease and PCV-13 serotype CAP in adults. A key strength of this study is the 293 identification of pneumococcal serotypes in non-bacteraemic cases of CAP. The main limitation

294 of the study was the inability to detect non-PCV-13 serotypes other than serotype 8 in patients 295 with non-bacteraemic CAP. For the comparative analysis, patients with untyped pneumococcal 296 CAP were considered to have non-PCV-13 serotype CAP. Although the Bio-plex assay has a high

297 sensitivity for the detection of 14 serotypes, some patients with untyped pneumococcal CAP may 298 have had PCV-13 serotype CAP; thus any differences identified between groups are likely to be 299 conservative for the association with PCV-13 serotype disease. The overall proportion of study 300 patients with dementia as a co-morbid illness was low in this study. Whilst this may be a true 301 finding, we are unable to exclude temporal selection bias given the high prevalence of PCV-7 302 serotypes and contemporaneous national data from the British Thoracic Society CAP audit which 303 demonstrated an increase in the proportions of patients with dementia over the study period 304 (the inverse of which was seen in the present study). ${ }^{40}$

306 For incidence calculations, population level data on influenza risk groups from 2015/16 were used

307 in place of pneumococcal clinical risk groups as a national registry of the latter is lacking. 308 Influenza risk groups overlap with pneumococcal clinical risk groups, with the exception of 309 cochlear implants and CSF leaks, the latter two of which had no study patients. The impact of this 310 limitation on study results is likely to be small. Completeness of case ascertainment and 
311 microbiological testing would also be expected to influence incidence calculations. A small

312 proportion of patients admitted over weekends and with very short lengths of stay may not have

313 been recruited to the study; urine samples were also not available for serotype analysis in 56

314 (8.7\%) patients with pneumococcal CAP. Therefore, incidence rate estimates are likely to be 315 conservative.

\section{Implications of results}

317 Whilst PCV-13 vaccine efficacy against VT serotype pneumococcal pneumonia has been

318 demonstrated in older adults, the effect of conjugate vaccine administration across other clinical

319 risk groups remains uncertain. ${ }^{17}$ An important question for pneumococcal vaccine policy is 320 whether there are identifiable groups of adults at risk of VT disease despite herd protection

321 effects arising from pneumococcal vaccination programmes. We found that in the presence of a

322 strong infant pneumococcal vaccination programme, the burden of PCV13 disease is greater in

323 adults outside the traditional 'at-risk' groups compared to adults in 'at-risk' groups. Adults in the

324 traditional 'at-risk' groups were more likely to be hospitalised with non-PCV-13 serotype CAP

325 than PCV-13 serotype CAP. Offering PCV-13 vaccination to adults in clinical risk groups may

326 therefore be of limited benefit in this setting. 


\section{Acknowledgments}

The authors would like to thank Tim Harrison from RVPBRU, Colindale for his support; Sally-Ann Nguyen, Christine More and Seyi Eletu from RVPBRU, Colindale and Robert Cave, Andrew Shelton, Adrian Patrick, Michelle Stannard and Joanne Palfreyman from the Microbiology Department, Nottingham University Hospitals for processing the urine samples; clinicians and staff of Nottingham University Hospitals NHS Trust, and Gemma Thompson, Emily Jarvis, Melanie Caine and Gaynor Bates for assisting with patient recruitment; Sarah Mayfield and Vanessa Macgregor from Public Health England for population data regarding clinical risk groups. The authors acknowledge the support of Alere in providing BinaxNOW test kits towards the conduct of this study. This study was funded by an investigator initiated unrestricted grant from Pfizer (formerly Wyeth).

\section{$\underline{\text { Author contributions }}$}

Study conception and design: PD, CT and WSL

Acquisition of data: CR, TB, SG and CS

Analysis and interpretation of data: PD, CT, TM and WSL

Drafting of manuscript: PD and WSL

Critical revision: all authors 


\section{Funding}

This study was funded by an investigator initiated unrestricted grant from Pfizer (formerly Wyeth).

\section{Conflicts of interest}

PD - received salaries derived from an investigator initiated unrestricted grant from Pfizer

CR - received salaries part funded by an NIHR grant and an investigator initiated unrestricted grant from Pfizer during his research

TB - received salaries derived from an investigator initiated unrestricted grant from Pfizer during his research

SG - received salaries derived from an unrestricted grant from Pfizer

TMM - nil

CS - nil

CT - received consulting payment from GSK in 2013 and an honorarium from Sanofi Pasteur in 2015

WSL-received grants from the National Institute of Health Research and an investigator initiated unrestricted grant from Pfizer. 


\section{Table and figures}


Table 1 - Distribution of co-morbid diseases in adults with pneumococcal CAP

\begin{tabular}{l|cc}
\hline & 16-64 years & $\geq 65$ years \\
& $n=266$ & $n=377$ \\
\hline Chronic heart disease & $14(5.3)$ & $110(29.2)$ \\
Chronic respiratory disease & $23(8.7)$ & $107(28.4)$ \\
Diabetes & $26(9.8)$ & $56(14.9)$ \\
Chronic kidney disease & $4(1.5)$ & $44(11.7)$ \\
Chronic liver disease & $6(2.3)$ & $4(1.1)$ \\
Immunosuppressed & $8(3.0)$ & $10(2.7)$ \\
Cancer & $12(4.5)$ & $26(6.9)$ \\
Dementia & $0(0.0)$ & $19(5.0)$ \\
Cerebrovascular disease & $5(1.9)$ & $62(16.5)$ \\
\hline
\end{tabular}

All values given as $n(\%)$ 
Table 2 - Clinical features of adults admitted with CAP and comparative analysis of those with PCV-13 versus non-PCV-13 serotype CAP

\begin{tabular}{|c|c|c|c|c|c|}
\hline & All cause CAP & \multicolumn{2}{|c|}{ Pneumococcal CAP } & \multirow[t]{2}{*}{ OR (95\%CI) } & \multirow[t]{2}{*}{$p$ value } \\
\hline \multirow[b]{2}{*}{ Age } & $(n=2224)$ & $\begin{array}{l}\text { PCV-13 disease } \\
\quad(n=337)\end{array}$ & $\begin{array}{c}\text { Non-PCV-13 } \\
\text { disease }(n=250)\end{array}$ & & \\
\hline & & & & & \\
\hline $16-49$ years & $431(19.4)$ & $86(25.5)$ & $58(23.2)$ & Reference & $0.216^{\dagger}$ \\
\hline $50-64$ years & $424(19.1)$ & $58(17.2)$ & $39(15.6)$ & $1.00(0.59-1.70)$ & \\
\hline $65-74$ years & $468(21.0)$ & $75(22.3)$ & $44(17.6)$ & $1.15(0.70-1.90)$ & \\
\hline $75-84$ years & 577 (25.9) & 67 (19.9) & $72(28.8)$ & $0.63(0.39-1.01)$ & \\
\hline$\geq 85$ years & $324(14.6)$ & $51(15.1)$ & $37(14.8)$ & $0.93(0.54-1.59)$ & \\
\hline Male & $1225(55.1)$ & $180(53.4)$ & $115(46.0)$ & $1.35(0.97-1.87)$ & 0.076 \\
\hline Care home resident ${ }^{¥}$ & $92(4.2)$ & $15(4.5)$ & $13(5.2)$ & $0.86(0.40-1.85)$ & 0.702 \\
\hline PPV23 vaccination $¥$ & $931(47.3)$ & $123(41.6)$ & $108(50.2)$ & $0.70(0.49-1.00)$ & 0.052 \\
\hline \multicolumn{6}{|l|}{ Smoking status ${ }^{¥}$} \\
\hline Never & $612(29.1)$ & $82(25.8)$ & $64(27.2)$ & Reference & $0.864^{\dagger}$ \\
\hline Ex & 989 (46.9) & $144(45.3)$ & $97(41.3)$ & $1.16(0.76-1.76)$ & \\
\hline Current & $506(24.0)$ & $92(28.9)$ & $74(31.5)$ & $0.97(0.62-1.52)$ & \\
\hline Alcohol excess & $47(2.1)$ & $9(2.7)$ & $7(2.8)$ & $0.95(0.35-2.60)$ & 0.924 \\
\hline Chronic respiratory disease & $572(25.7)$ & $52(15.4)$ & $66(26.4)$ & $0.51(0.34-0.77)$ & 0.001 \\
\hline Asthma & $267(12.0)$ & 40 (11.9) & $39(15.6)$ & $0.73(0.45-1.17)$ & 0.191 \\
\hline COPD & 509 (22.9) & $46(13.7)$ & $60(24.0)$ & $0.50(0.33-0.77)$ & 0.001 \\
\hline Chronic heart disease & $500(22.5)$ & $63(18.7)$ & $53(21.2)$ & $0.85(0.57-1.29)$ & 0.451 \\
\hline CCF & $146(6.6)$ & $19(5.6)$ & $15(6.0)$ & $0.94(0.47-1.88)$ & 0.853 \\
\hline IHD & $249(11.2)$ & $28(8.3)$ & $27(10.8)$ & $0.75(0.43-1.31)$ & 0.306 \\
\hline Diabetes & 305 (13.7) & $48(14.2)$ & $26(10.4)$ & $1.43(0.86-2.38)$ & 0.166 \\
\hline Chronic liver disease & $24(1.1)$ & $5(1.5)$ & $5(2.0)$ & $0.74(0.21-2.58)$ & 0.751 \\
\hline Chronic kidney disease & $169(7.6)$ & $17(5.0)$ & $27(10.8)$ & $0.44(0.23-0.83)$ & 0.009 \\
\hline Immunocompromised & $82(3.7)$ & $6(1.8)$ & $8(3.2)$ & $0.55(0.19-1.60)$ & 0.265 \\
\hline
\end{tabular}




\begin{tabular}{|c|c|c|c|c|c|}
\hline Severely immunocompromised* & $22(1.0)$ & $0(0.0)$ & $5(2.0)$ & - & - \\
\hline Active malignancy & $169(7.6)$ & $21(6.2)$ & $13(5.2)$ & $1.21(0.59-2.47)$ & 0.597 \\
\hline Dementia & $45(2.0)$ & $16(4.8)$ & $3(1.2)$ & $4.10(1.17-14.34)$ & 0.016 \\
\hline Cerebrovascular disease & $202(9.1)$ & $42(12.5)$ & $23(9.2)$ & $1.41(0.82-2.41)$ & 0.213 \\
\hline \multicolumn{6}{|l|}{ Number of clinical risk factors: } \\
\hline 0 & $1078(48.5)$ & $198(58.8)$ & $119(47.6)$ & Reference & $0.016^{\dagger}$ \\
\hline 1 & $751(33.8)$ & $98(29.1)$ & $92(36.8)$ & $0.64(0.44-0.92)$ & \\
\hline 2 & $289(13.0)$ & $30(8.9)$ & $26(10.4)$ & $0.69(0.39-1.23)$ & \\
\hline$\geq 3$ & $106(4.8)$ & $11(3.3)$ & $13(5.2)$ & $0.51(0.22-1.18)$ & \\
\hline Low severity (CURB65 $\leq 1$ ) & $1029(46.3)$ & $134(39.8)$ & $100(40.0)$ & Reference & $0.732^{+}$ \\
\hline Moderate severity (CURB65=2) & $684(30.8)$ & $107(31.8)$ & $84(33.6)$ & $0.95(0.65-1.40)$ & \\
\hline High severity (CURB65 $\geq 3$ ) & $511(23.0)$ & $96(28.5)$ & $66(26.4)$ & $1.09(0.72-1.63)$ & \\
\hline 30-day mortality & $230(10.3)$ & $28(8.3)$ & $19(7.6)$ & $1.10(0.60-2.02)$ & 0.755 \\
\hline
\end{tabular}

All values expressed as $\mathrm{n}(\%) ; \mathrm{t}-\mathrm{p}$ for trend; ${ }^{*}$ - care home and smoking status unavailable for 26 and 117 patients, respectively.

PPV23 - 23-valent pneumococcal polysaccharide vaccine ( ${ }^{*}$ data unavailable for 254 patients), COPD - chronic obstructive pulmonary disease, CCF - congestive cardiac failure, IHD - ischaemic heart disease. * - severely immunocompromised group consists of bone marrow transplant patients, patients with acute and chronic leukaemia, multiple myeloma or those with genetic disorders affecting the immune system

OR and $p$ values compare PCV-13 serotype disease to non-PCV-13 disease. 
Table 3 - Association between clinical risk group and PCV-13 disease

\begin{tabular}{l|cc}
\hline & aOR $(95 \% \mathrm{CI})$ & $p$ value \\
\hline $\begin{array}{l}\text { 16-64 yrs with no clinical risk } \\
\text { factor }\end{array}$ & Reference & - \\
$\begin{array}{l}\text { 16-64 yrs with clinical risk } \\
\text { factor(s) }\end{array}$ & $0.58(0.32-1.06)$ & 0.077 \\
$\geq 65$ yrs with no clinical risk \\
factor \\
$\geq 65$ yrs with clinical risk \\
$\begin{array}{l}\text { factor(s) } \\
\text { Male gender }\end{array}$ & $0.98(0.61-1.55)$ & 0.915 \\
\hline
\end{tabular}

Table 4 - Association between pneumococcal serotypes and individuals at risk of pneumococcal disease

\begin{tabular}{c|cccc}
\hline Serotype & $\begin{array}{c}\text { 'At-risk' group } \\
(n=403)\end{array}$ & $\begin{array}{c}\text { No risk group } \\
(n=184)\end{array}$ & OR (95\%Cl) & P value \\
\hline 1 & 34 & 28 & $0.78(0.39-1.57)$ & 0.485 \\
3 & 21 & 3 & $4.50(1.22-16.56)$ & $\mathbf{0 . 0 2 4}$ \\
4 & 9 & 7 & $0.83(0.28-2.48)$ & 0.734 \\
5 & 20 & 4 & $3.21(0.99-10.43)$ & $\mathbf{0 . 0 5 2}$ \\
$6 \mathrm{~A} / \mathrm{C}$ & 19 & 4 & $3.05(0.94-9.95)$ & 0.064 \\
$7 \mathrm{~F} / \mathrm{A}$ & 25 & 44 & $0.37(0.18-0.73)$ & $\mathbf{0 . 0 0 4}$ \\
8 & 42 & 27 & Reference & \\
14 & 41 & 9 & $2.93(1.23-6.98)$ & $\mathbf{0 . 0 1 5}$ \\
$19 \mathrm{~A}$ & 27 & 11 & $1.58(0.67-3.70)$ & 0.294 \\
\hline
\end{tabular}

All values given as n; 'At-risk' group defined as those aged 16-64 years with clinical risk factors for pneumococcal disease or those aged $\geq 65$ years 
Figure 1 - Gender and age adjusted odds of PCV-13 serotype CAP with increasing numbers of clinical risk factors for pneumococcal infection

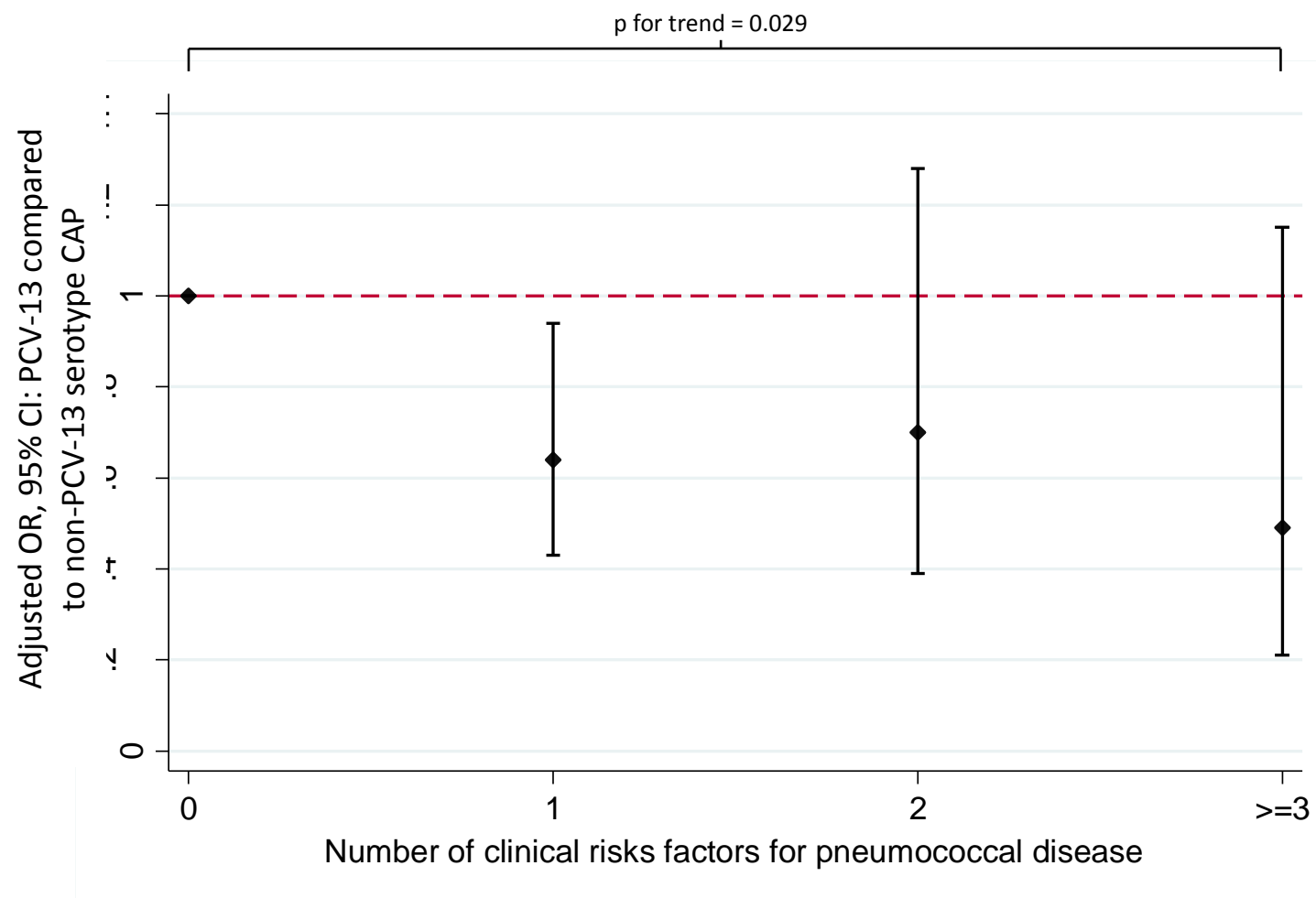

- OR $\longmapsto$ Upper Cl (95\%)/Lower Cl (95\%) 
Figure 2 - Incidence of PCV-13 and non-PCV-13 pneumococcal CAP by age and clinical risk group

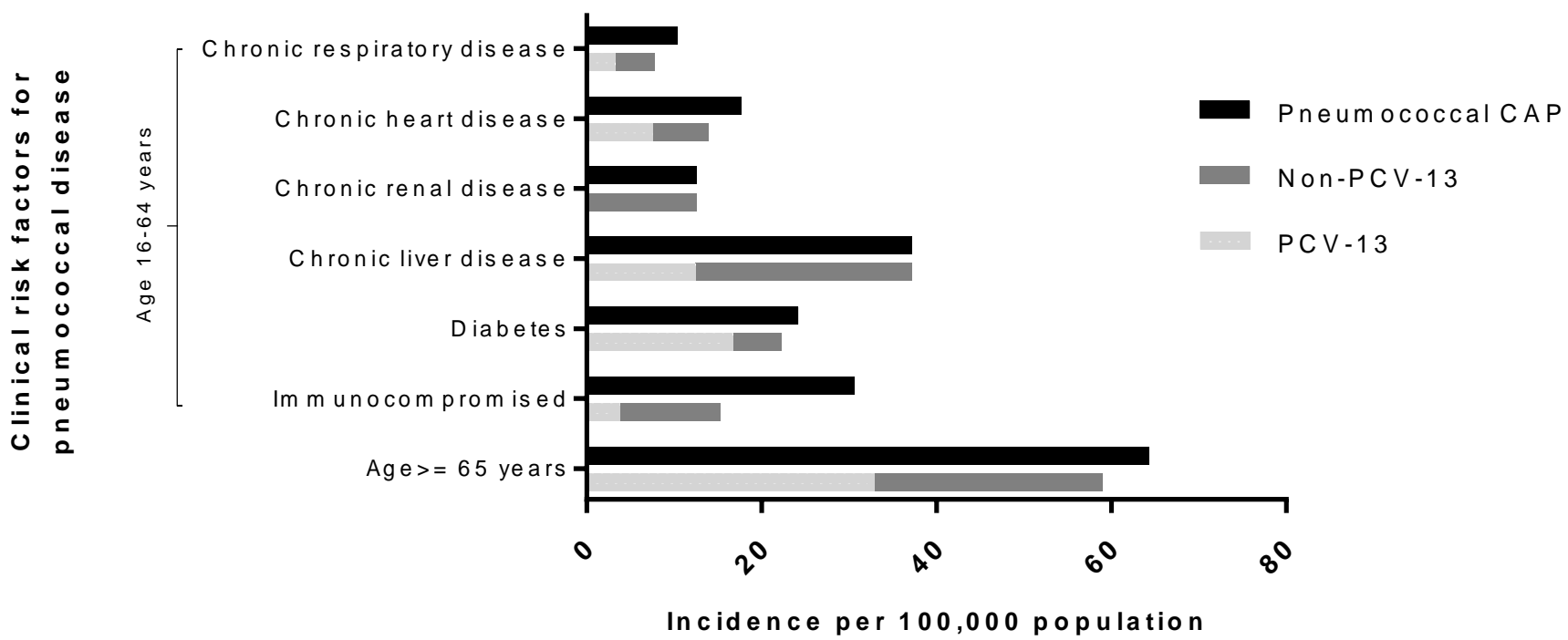




\section{References}

1. Nuorti JP, Butler JC, Farley MM, et al. Cigarette smoking and invasive pneumococcal disease. Active Bacterial Core Surveillance Team. N Engl J Med 2000;342(10):681-9.

2. Lipsky BA, Boyko EJ, Inui TS, et al. Risk factors for acquiring pneumococcal infections. Arch Intern Med 1986;146(11):2179-85.

3. van Hoek AJ, Andrews N, Waight PA, et al. The effect of underlying clinical conditions on the risk of developing invasive pneumococcal disease in England. J Infect 2012;65(1):17-24.

4. Vila-Corcoles A, Aguirre-Chavarria C, Ochoa-Gondar O, et al. Influence of chronic illnesses and underlying risk conditions on the incidence of pneumococcal pneumonia in older adults. Infection 2015;43(6):699-706.

5. Naucler P, Darenberg J, Morfeldt E, et al. Contribution of host, bacterial factors and antibiotic treatment to mortality in adult patients with bacteraemic pneumococcal pneumonia. Thorax 2013.

6. Klemets $\mathrm{P}$, Lyytikainen $\mathrm{O}$, Ruutu $\mathrm{P}$, et al. Invasive pneumococcal infections among persons with and without underlying medical conditions: implications for prevention strategies. $B M C$ Infect Dis 2008;8:96.

7. Public Health England. Immunisation against Infectious Disease, 2013:295-314.

8. Moberley SA, Holden J, Tatham DP, et al. Vaccines for preventing pneumococcal infection in adults. Cochrane Database Syst Rev 2008(1):CD000422.

9. Huss $A$, Scott $P$, Stuck AE, et al. Efficacy of pneumococcal vaccination in adults: a meta-analysis. CMAJ : Canadian Medical Association Journal 2009;180(1):48-58.

10. Moore RA, Wiffen PJ, Lipsky BA. Are the pneumococcal polysaccharide vaccines effective? Meta-analysis of the prospective trials. BMC Fam Pract 2000;1:1-1.

11. Jackson LA, Neuzil KM, Yu O, et al. Effectiveness of Pneumococcal Polysaccharide Vaccine in Older Adults. N Engl J Med 2003;348(18):1747-55.

12. Diao WQ, Shen N, Yu PX, et al. Efficacy of 23-valent pneumococcal polysaccharide vaccine in preventing community-acquired pneumonia among immunocompetent adults: A systematic review and meta-analysis of randomized trials. Vaccine 2016;34(13):1496-503.

13. Feikin $D R$, Elie $C M$, Goetz $M B$, et al. Randomized trial of the quantitative and functional antibody responses to a 7-valent pneumococcal conjugate vaccine and/or 23-valent polysaccharide vaccine among HIV-infected adults. Vaccine 2001;20(3-4):545-53.

14. Dransfield MT, Nahm MH, Han MK, et al. Superior immune response to protein-conjugate versus free pneumococcal polysaccharide vaccine in chronic obstructive pulmonary disease. Am J Respir Crit Care Med 2009;180(6):499-505.

15. de Roux A, Schmole-Thoma B, Siber GR, et al. Comparison of pneumococcal conjugate polysaccharide and free polysaccharide vaccines in elderly adults: conjugate vaccine elicits improved antibacterial immune responses and immunological memory. Clin Infect Dis 2008;46(7):1015-23.

16. French N, Gordon SB, Mwalukomo T, et al. A trial of a 7-valent pneumococcal conjugate vaccine in HIV-infected adults. N Engl J Med 2010;362(9):812-22. 
17. Bonten MJM, Huijts SM, Bolkenbaas $M$, et al. Polysaccharide Conjugate Vaccine against Pneumococcal Pneumonia in Adults. N Engl J Med 2015;372(12):1114-25.

18. Miller E, Andrews NJ, Waight PA, et al. Herd immunity and serotype replacement 4 years after seven-valent pneumococcal conjugate vaccination in England and Wales: an observational cohort study. Lancet Infect Dis 2011;11(10):760-8.

19. Moore CE, Paul J, Foster D, et al. Reduction of invasive pneumococcal disease 3 years after the introduction of the 13-valent conjugate vaccine in the Oxfordshire region of England. $J$ Infect Dis 2014;210(7):1001-11.

20. Waight PA, Andrews NJ, Ladhani SN, et al. Effect of the 13-valent pneumococcal conjugate vaccine on invasive pneumococcal disease in England and Wales 4 years after its introduction: an observational cohort study. Lancet Infect Dis 2015;15(5):535-43.

21. Rodrigo C, Bewick T, Sheppard C, et al. Impact of infant 13-valent pneumococcal conjugate vaccine on serotypes in adult pneumonia. Eur Respir J 2015;45(6):1632-41.

22. Sheppard CL, Harrison TG, Smith MD, et al. Development of a sensitive, multiplexed immunoassay using XMAP beads for detection of serotype-specific streptococcus pneumoniae antigen in urine samples. J Med Microbiol 2011;60(Pt 1):49-55.

23. Sinclair A, Xie X, Teltscher $M$, et al. Systematic Review and Meta-Analysis of a Urine-Based Pneumococcal Antigen Test for Diagnosis of Community-Acquired Pneumonia Caused by Streptococcus pneumoniae. J Clin Microbiol 2013;51(7):2303-10.

24. Rozenbaum $\mathrm{MH}$, van Hoek AJ, Fleming $D$, et al. Vaccination of risk groups in England using the 13 valent pneumococcal conjugate vaccine: economic analysis. BMJ : British Medical Journal 2012;345:e6879.

25. Office for National Statistics. 2011 Census. Census 2011: Usual resident population by single year of age, unrounded estimates, local authorities in the United Kingdom. https://www.ons.gov.uk/peoplepopulationandcommunity/populationandmigration/pop ulationestimates/datasets/2011censuspopulationestimatesbysingleyearofageandsexforl ocalauthoritiesintheunitedkingdom

26. Wagenvoort GH, Knol MJ, de Melker HE, et al. Risk and outcomes of invasive pneumococcal disease in adults with underlying conditions in the post-PCV7 era, The Netherlands. Vaccine 2016;34(3):334-40.

27. Browall S, Backhaus $E$, Naucler $P$, et al. Clinical manifestations of invasive pneumococcal disease by vaccine and non-vaccine types. Eur Respir J 2014;44(6):1646-57.

28. Lujan M, Burgos J, Gallego M, et al. Effects of immunocompromise and comorbidities on pneumococcal serotypes causing invasive respiratory infection in adults: implications for vaccine strategies. Clin Infect Dis 2013;57(12):1722-30.

29. Millar EV, Watt JP, Bronsdon MA, et al. Indirect effect of 7-valent pneumococcal conjugate vaccine on pneumococcal colonization among unvaccinated household members. Clin Infect Dis 2008;47(8):989-96.

30. van Hoek AJ, Sheppard CL, Andrews NJ, et al. Pneumococcal carriage in children and adults two years after introduction of the thirteen valent pneumococcal conjugate vaccine in England. Vaccine 2014;32(34):4349-55.

31. Muhammad RD, Oza-Frank R, Zell E, et al. Epidemiology of invasive pneumococcal disease among high-risk adults since the introduction of pneumococcal conjugate vaccine for children. Clin Infect Dis 2013;56(5):e59-67. 
32. Lexau CA, Lynfield R, Danila $R$, et al. Changing epidemiology of invasive pneumococcal disease among older adults in the era of pediatric pneumococcal conjugate vaccine. JAMA 2005;294(16):2043-51.

33. Cabaj JL, Nettel-Aguirre A, MacDonald J, et al. Influence of Childhood Pneumococcal Conjugate Vaccines on Invasive Pneumococcal Disease in Adults With Underlying Comorbidities in Calgary, Alberta (2000-2013). Clin Infect Dis 2016;62(12):1521-6.

34. Harboe ZB, Dalby T, Weinberger DM, et al. Impact of 13-valent pneumococcal conjugate vaccination in invasive pneumococcal disease incidence and mortality. Clin Infect Dis 2014;59(8):1066-73.

35. Brueggemann $A B$, Griffiths DT, Meats $E$, et al. Clonal relationships between invasive and carriage Streptococcus pneumoniae and serotype- and clone-specific differences in invasive disease potential. J Infect Dis 2003;187(9):1424-32.

36. Sjostrom K, Spindler C, Ortqvist A, et al. Clonal and capsular types decide whether pneumococci will act as a primary or opportunistic pathogen. Clin Infect Dis 2006;42(4):451-9.

37. Jansen AGSC, Rodenburg GD, van der Ende A, et al. Invasive Pneumococcal Disease among Adults: Associations among Serotypes, Disease Characteristics, and Outcome. Clin Infect Dis 2009;49(2):e23-e29.

38. Bewick T, Sheppard C, Greenwood S, et al. Serotype prevalence in adults hospitalised with pneumococcal non-invasive community-acquired pneumonia. Thorax 2012;67(6):540-5.

39. Rodrigo C, Bewick T, Sheppard C, et al. Clinical features of adults with seven-valentconjugated-vaccine-serotype pneumococcal pneumonia. Vaccine 2014;32(13):1460-5.

40. Daniel P, Woodhead M, Welham S, et al. Mortality reduction in adult community-acquired pneumonia in the UK (2009-2014): results from the British Thoracic Society audit programme. Thorax 2016;71(11):1061-63. 